\title{
As Relações entre Gamificação, Padrões de Interface e Mobilidade no Desenvolvimento de Aplicações Educacionais
}

\author{
Cristiane Ellwanger, Cristina Paludo Santos, Guilherme J. Moreira \\ Universidade Regional Integrada do Alto Uruguai e das Missões (URI) 98.802-470 - \\ Santo Ângelo - RS - Brasil \\ cristianeellwanger@gmail.com, paludo@santoangelo.uri.br, \\ guilhermejm@hotmail.com
}

\begin{abstract}
This paper demonstrates the development of an additional module, aimed at educational environments that favors interaction and motivation of users to use the system, developed under the Android platform. As a result, the module also presents itself as not only benefit the end users (teachers and students), but the computer professionals, as they provide a theoretical and practical foundation to behold requirements gamification integrated the use of interface standards specifically for applications in Android..

Resumo. O presente artigo demonstra o desenvolvimento de um recurso que favorece a interação, motivando usuários para o uso de sistemas direcionados a contextos educacionais, sob a plataforma Android. Como resultados, o módulo adicional desenvolvido oferece subsídios não somente para os usuários finais (educadores e educandos), mas também a profissionais da computação, por fornecer um embasamento teórico-prático de como contemplar requisitos de gamificação agregados ao uso de padrões de interface em aplicações desenvolvidas em Android.
\end{abstract}

\section{Introdução}

A gamificação oferece contribuições a ambientes educacionais ao agregar novas formas de motivar educandos na realização de tarefas cotidianas, entretanto há certa dificuldade em unir os conceitos de gamificação ao uso de dispositivos móveis, devido à falta de padrões de interfaces para desenvolvimento de aplicações neste tipo de dispositivo bem como às limitações atreladas ao desenvolvimento destas pelas próprias características físicas dos dispositivos móveis [Ellwanger, Santos e Maciel, 2013][Mejia, 2013].

Ambientes educacionais constituem-se de experiências gameficadas ao se atribuir pontuações a educandos nas diferentes tarefas por eles realizadas, mesmo que procedimento não possibilite ao aluno uma profunda imersão no contexto da tarefa. Em contrapartida, videogames e mundos virtuais destacam-se por obter/reter a atenção e envolvimento de seus usuários [Lee apud McGonigal 2011], ou seja, as pontuações atribuídas integradas a uma série de outros recursos de provenientes de ambientes digitais é o que proporciona esta imersão.

Assim a gamificação oferece a projetos, voltados ao processo de ensino e de aprendizagem, a possibilidade de se integrar o uso de regras, fatores emocionais e papéis sociais integrados a realização de uma determinada tarefa por parte do educando. Ao seguir as regras pré-estabelecidas pelo ambiente estudantes. Além disso, regras previamente determinadas possibilita aos estudantes estruturar e reestruturar seu próprio aprendizado para uma melhor compreensão das atividades que lhes são propostas e se apresentam como recursos adicionais quando utilizados em ambientes virtuais de aprendizagem [Lee, 2011]. 
Neste contexto, este artigo apresenta a integração de um módulo adicional ao modelo MobilEduc [Ellwanger, Santos e Maciel, 2013], desenvolvido de forma a contemplar conceitos de gamificação e a utilização de padrões de desenvolvimento de interfaces específicas para aplicações Android, no intuito não somente de adequar o modelo às novas tecnologias, mas também como forma oferecer subsídios motivacionais a educandos para a realização de atividades bem como demonstrar como padrões de interface foram integrados ao modelo. Sua contribuição não está nas técnicas de gamificação ou nos padrões utilizados para contemplar o modelo, mas sim no processo de integração das mesmas.

\section{Gamificação e Padrões de Interface Agregadas ao Modelo MobilEduc - Aspectos Metodológicos}

O modelo MobilEduc foi desenvolvido no intuito de orientar o desenvolvimento de aplicações direcionadas ao processo de ensino e aprendizagem em dispositivos móveis, mais especificamente, telefones celulares ou smartphones, agregando tanto preceitos de AVAs quanto de M-Learning [Maciel 2012] [Ellwanger, Santos e Maciel 2013]. Os eixos iniciais contemplados no modelo relacionam-se ao processo de ensino e aprendizagem em dispositivos móveis (M-Learning).

A evolução do modelo foi implementada com vistas à incorporação de um módulo adicional no intuito de agregar as características específicas de gamificação e a atribuição de padrões de desenvolvimento de interfaces com foco em aplicações para Android, a fim de proporcionar novas formas de interação e de execução de tarefas por parte do usuário. Para isso foram utilizados os conceitos de Deterding (2011) para aplicação de estratégias de gamificação e os pressupostos de Nudelman (2013) no que se refere ao desenvolvimento de padrões interfaces em aplicações Android. Assim, para a agregação deste módulo ao modelo foi organizada no intuito de se contemplar os seguintes parâmetros:

a) Estratégias de gamificação - Envolve as estratégias utilizadas no sistema. As principais estratégias de gamificação apresentadas no modelo, mas não limitado a estas, são: Achievements: Pontuação/Ranking competitivo e Experiência/Niveis de evolução pessoal.

b) Padrões de Projeto - Contempla quesitos relacionados a padrões para uma melhor adaptação do usuário ao sistema e a percepção dos elementos presentes na interface.

c) Eixo de Produção Gameficado - Refere-se às formas de criação/inclusão da gamificação na etapa de desenvolvimento de atividades por parte do educador.

d) Eixo de Documentação/Informação Gameficado - Define os meios de prover as atividades e materiais pedagógicos, bem como o feedback das atividades gameficadas. Define ainda a forma de apresentar os recursos internos do sistema (ranking, status pessoal do aluno/usuário).

e) Forma de Apresentação da Gamificação - Refere-se às formas de apresentar a gamificação e seus conceitos nas atividades pedagógicas.

A partir do modelo geral de agregação da gamificação, foram definidas etapas para o desenvolvimento do sistema, as quais foram referenciadas como: design, 
desenvolvimento e testes e resultados. A etapa de Design consiste na decisão de quais estratégias de gamificação são adotadas e quais são os padrões de projeto de interface necessários para contemplá-las. A etapa de desenvolvimento envolve a aplicação das estratégias de gamificação definidas na etapa de design, no desenvolvimento dos meios de produção das atividades e materiais pedagógicos, na verificação de como as atividades e materiais serão apresentados aos usuários, bem como o momento adequado para o feedback da gamificação. Já a etapa de testes e resultados refere-se a realização de testes funcionais, a partir da realização de atividades gameficadas, as quais são retratadas ao usuário.

A aplicação foi desenvolvida sob a plataforma Android. As estratégias de gamificação foram aplicadas a partir da implantação de técnicas específicas para este fim, considerando a forma como os dados são armazenados e referenciam os resultados. No que se refere aos padrões de projeto foram considerados os preceitos definidos por Nudelman (2013), visando a estruturação dos elementos gameficados e uma melhor visualização da interface do sistema. Na composição dos padrões contemplou-se os padrões de navegação referenciados como canivete suíço, hot zones, entrada de texto livre, toast alert e alerta sobreposto (o modelo não necessariamente limita-se a estes). Uma atenção especial é necessária para que sejam evitados os chamados anti-padrões, os quais retratam, de forma generalizada, os erros mais comuns no desenvolvimento de aplicações móveis, contrariando preceitos voltados à usabilidade geral do sistema, conforme demonstra a Figura 1.

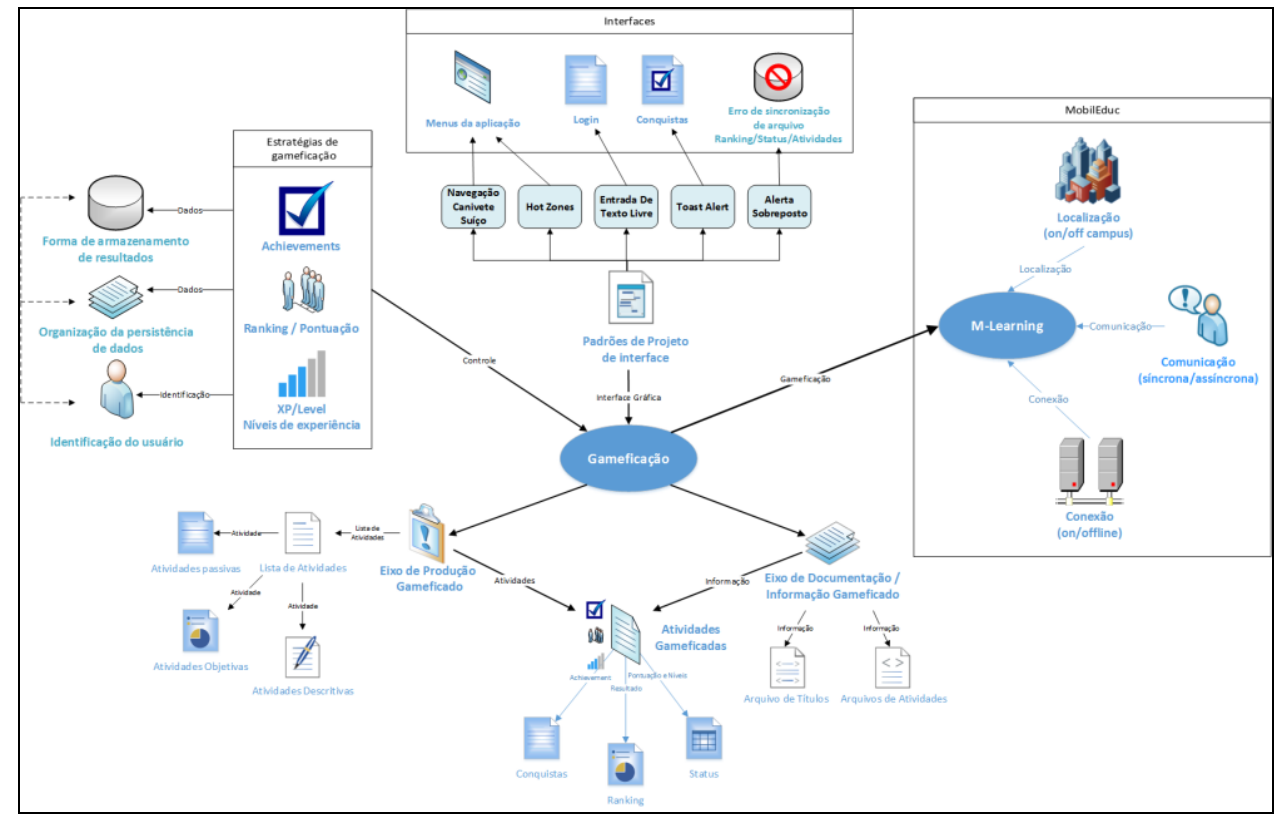

Figura 1. Forma de estruturação do módulo adicional.

O eixo "Produção Gameficado" envolve o desenvolvimento de atividades e materiais direcionados a aprendizagem. Essas atividades são cadastradas no sistema pelo professor, a partir de dois arquivos de texto. Um deles contém os títulos de cada atividade que dão origem a uma lista de botões na interface, enquanto o outro arquivo é necessário para a composição de cada atividade, em que o controle da leitura do arquivo é feito a partir de cada linha, sendo definido um botão na lista de atividades para cada linha com seu respectivo título. O controle de leitura do arquivo de cada atividade é 
feito com adição de caracteres especiais (@, \&, \%, \#, * e \$). O símbolo “@” define o número de questões corretas para a obtenção de um achievement, "\&" representa a pontuação atribuída a cada questão correta, "\%" define o valor de experiência recebida pelo aluno após a conclusão da atividade, “\#” representa uma determinada questão, para que no momento de leitura do arquivo seja criada uma "TextView" com a questão correspondente, "**"define uma questão incorreta, criando um "RadioButton" com a mesma e por fim, o símbolo" \$" representa a resposta correta da questão correspondente, sendo definida também como um "RadioButton", em conjunto com as outras questões correspondentes dentro de um RadioGroup. Após o cadastro das atividades no sistema por meio do eixo de produção, essas atividades são recuperadas através da leitura dos arquivos com as marcações de definição dos componentes e representam o eixo Documentação/Informação Gameficado.

\section{Resultados advindos do Módulo Adicional}

Para a implementação do módulo adicional foram considerados os eixos correspondentes a padrões de projeto de interface, estratégias de gamificação, produção, documentação/informação e atividades, respectivamente.

O eixo de Padrões de Projeto de Interface define a implementação e o uso de padrões de projeto no modelo e favorece o uso de técnicas de gamificação, bem como o desenvolvimento do sistema como um todo; o eixo de Estratégias de Gamificação compreende a definição das estratégias de gamificação utilizadas e a definição da forma de representação dos dados oriundos dessas técnicas, bem como o tratamento para seu armazenamento e utilização posterior; o Eixo de Produção Gameficado representa a forma de desenvolvimento das atividades educacionais considerando a gamificação na produção destas, bem como a sua organização no ambiente de aprendizagem através da forma de exibição e acesso de cada atividade e material pedagógico disponibiliado; o Eixo de Documentação/Informação Gameficado aborda a forma de construção das atividades e as tecnologias necessárias para que estas contemplem a gamificação e, por fim, o eixo Atividades Gameficadas contempla a forma de representação dos resultados da gamificação para com o usuário, provendo o feedback do uso dessas técnicas.

Diante do exposto, a gamificação é a convergência central de todas as diretrizes da expansão do modelo MobilEduc, visto que está diretamente vinculada ao núcleo $M$ Learning bem como aos eixos de Localização, Comunicação e Conexão, provenientes do mesmo[Maciel, 2012]. Logo, o módulo adicional se volta a uma aplicação para smartphones Android, constituindo-se de um ambiente virtual que propicia a aprendizagem.

\section{Conclusões e Direcionamentos para Trabalhos Futuros}

A utilização de técnicas de gamificação no âmbito educacional traz grandes benefícios para os educadores, pois proporciona a aplicação uma grande variedade de estratégias para motivar os alunos à participação e ao engajamento no processo de ensino e de aprendizagem. No que tange aos educandos, benefícios diretos são advindos a partir do conhecimento adquirido em uma plataforma disponível independentemente de horário ou local, para um maior envolvimento nas atividades por eles realizadas. 
A partir do presente trabalho foi possível definir o modelo para agregação de gamificação a ambientes de ensino e de aprendizagem em dispositivos móveis, bem como definir as diretrizes para o uso de padrões de projeto a fim de permitir uma melhor interação do usuário para com tais sistemas, bem como as respostas esperadas da utilização destas técnicas. Assim, o módulo adicional desenvolvido oferece subsídios não somente para os usuários finais (educadores e educandos), mas também aos próprios profissionais da área da computação, à medida que fornece um embasamento teórico-prático de como contemplar requisitos de gamificação agregados ao uso de padrões de interface em aplicações desenvolvidas em Android.

O uso do protótipo possibilitou a realização de uma avaliação preliminar e, embora dotado de uma boa aceitação pelos usuários e apresentando uma correta compreensão de seu funcionamento, ainda há a necessidade de se fazer testes de interação mais específicos, tanto no contexto de educandos quanto educadores, no intuito de se obter a melhoria contínua do protótipo desenvolvido. Além disso, os trabalhos futuros envolvem também o estudo e aplicação de estratégias de acessibilidade em dispositivos móveis bem como o acoplamento de outras funcionalidades específicas a tais dispositivos como o uso de tecnologias de geolocalização, as quais podem ser utilizadas como suporte à gamificação.

\section{Referências Bibliográficas}

Dantas, A. R., Veronese, G. O., Correa, A. L., Xavier, J. R., \& Werner, C. M. L. (2002). Suporte a Padrões no Projeto de Software. Caderno de Ferramentas do XVI Simpósio Brasileiro de Engenharia de Software, Gramado, Rio Grande do Sul, Brasil, Outubro.

Deterding, S., Dixon, D., Khaled, R., \& Nacke, L. From game design elements to gamefulness: Defining "gamification". MindTrek '11 Proceedings of the 15th International Academic MindTrek Conference: Envisioning Future Media Environments. 9-15. (2011).

Ellwanger, C.; Santos, C. P.; Maciel, G. J. M.; (2013). Concepção de Materiais Pedagógicos e o Estabelecimento de Situações de Aprendizagem em Dispositivos Móveis. In: Anais do XXXIII Congresso da Sociedade Brasileira de Computação: DesafIE! - II Workshop de Desafios da Computação Aplicada à Educação, pg 1310-1318, 2013.

Lee, J. J. \& Hammer, J. Gamification in Education: What, How, Why Bother? Academic Exchange Quarterly, 15(2). (2011).

Maciel, G. J. M. MobilEduc: Um Modelo para o Processo Ensino-Aprendizagem em Dispositivos Móveis. Trabalho de conclusão (2012). URI, Santo Ângelo, 2012.

McGonigal, J. Reality Is Broken: Why Games Make Us Better and How They Can Change the World. Penguin Press. New York, NY. (2011).

Mejia, Juan, "Impact of Gamification and Shared Situated Displays on Smartphone Application Engagement". Technical Library. Paper 152. (2013)

Moreno-Ger, P., Burgos, D., Martínez-Ortiz, I., Sierra, J. L., Fernández-Manjón, B., 2008. Educational game design for online education. Computers in Human Behavior. N. 24. Elsevier.

Nudelman, Greg. Padrões de Projeto para o Android. ISBN: 978-857522-358-1. Novatec Editora Ltda, (2013). 